\title{
Semantic/pragmatic factors affecting the salience of transfer verb arguments
}

\author{
Meghan M. Salomon \& Gregory Ward*
}

\begin{abstract}
Rohde et al. (2006) showed that, for transfer verbs, the salience of the RECIPIENT argument is a function of the particular COHERENCE RELATION posited by participants. Using a priming paradigm with transfer verbs and occupation-denoting NPs in isolated sentences, we find that participants are sensitive to the lexical properties of the transfer verb itself as measured by the relative salience of the associated transfer verb arguments. Alternatively, using a sentence completion paradigm - a more strategic task in which participants are guided by discourse-level features - we find participants are sensitive instead to the coherence relations of the relevant event, replicating Rohde et al. (2006). Our findings support the notion that coherence relations drive the interpretation of multi-sentence discourses while sentences considered in isolation are guided by particular features of the linguistic context.
\end{abstract}

Keywords. Lexical semantics, argument structure, coherence relations, psycholinguistics

1. Introduction. Language is a fundamental tool that people use to communicate and glean meanings, some of which are explicitly conveyed while others of which are only conveyed implicitly (Bolinger 1968; Grice 1970; Whorf 1956). Within research on sentence comprehension and text processing, various studies across various frameworks have shown that people rely on a remarkable range of semantic features to make predictions about an unfolding discourse (Graesser, Singer, and Trabasso 1994; Kintsch 1994; Kintsch and Van Dijk 1978). One such feature has to do with the argument structure of verbs and their corresponding thematic roles. It has been shown, for example, that when readers process sentences containing verbs that specify AGENT and PATIENT thematic roles, as in (1), AGENT arguments are generally found to be more salient than PATIENT arguments (Crawley, Stevenson, and Kleinman 1990; Gernsbacher and Hargreaves 1988; Wunderlich, 2006).

(1) The doctor smiled at the receptionist. AGENT PATIENT

However, with other verb classes with different argument structures, we find a different assignment of salience. Specifically, the class of so-called Transfer-of-Possession (ToP) verbs has been shown to affect the hearer's (or reader's) attention. Consider (2):

(2) The doctor handed a letter to the receptionist. SOURCE

Here, the doctor represents the SOURCE argument, i.e. the source of the letter being handed to the receptionist, who in turn represents the RECIPIENT argument, i.e. the recipient of the letter in question. Recent work on ToP verbs has shown a different pattern of thematic role salience than

\footnotetext{
*This work was supported by the US Department of Education, Institute of Education Sciences, Multidisciplinary Program in Education Sciences, Grant Award \#R305B140042. The conclusions, opinions, and other statements in this presentation are the authors and not necessarily those of the sponsoring institution. Authors: Meghan M. Salomon, Department of Psychology, Northwestern University (msalomon@u.northwestern.edu) \& Gregory Ward, Department of Linguistics, Northwestern University (gw@ northwestern.edu).
} 
the AGENT/PATIENT pattern discussed in connection with (1) above (Ferretti et al. 2009; Rohde, Kehler, and Elman 2006). Specifically, Rohde et al. have found that, when presented with ToP verbs, readers redirect their attention from the SOURCE role to the RECIPIENT role, rendering the latter more salient. Using a sentence-completion task, Rohde et al. 2006 found that verb type affects the interpretation of ambiguous pronouns with ToP verbs, as in (3):

(3) Steve threw a frisbee to Robert. He....
SOURCE

Participants were more likely to interpret the ambiguous pronoun beginning the subsequent sentence as specifying Robert. However, with non-ToP verbs, as in (1), the effect disappears, suggesting an inhibition of this attentional shift.

Within the class of ToP verbs, Rohde et al. (2006) distinguished three types based on two factors: (1) whether the transfer is GUARANTEED and (2) whether participants involved in the event are CO-LOCATED. A transfer is 'guaranteed' if the recipient must take actual possession of the object in question; otherwise the transfer is 'non-guaranteed'. Participants are 'co-located' if they are situated in close physical proximity. These two factors create three classes of ToP verbs, as illustrated in Table 1.1

\begin{tabular}{cccc} 
ToP Verb Type & Transfer Guaranteed & Participants Co-Located & Examples \\
\hline Type I & Yes & Yes & carry, bring, hand \\
Type II & No & Yes & kick, throw, lob \\
Type III & No & No & send, transport, ship \\
\hline
\end{tabular}

Table 1: ToP Verb Types

Type I ToP verbs are those that have a guaranteed transfer and the participants involved in the transfer are located in close proximity of one another (e.g. carry, bring, hand). Type II transfer verbs are ones for which the transfer is non-guaranteed, but the participants are nonetheless colocated (e.g. kick, throw, lob). The final type of transfer verbs, Type III, are ToP verbs whose associated transfers are not guaranteed, nor are the participants co-located (e.g. send, transport, ship). Using these three types of ToP verbs, Rohde et al. found that participants produced more references to the SOURCE argument than to the RECIPIENT argument only with Type III ToP verbs; surprisingly, they found no effect with Types I or II. ${ }^{2}$ This indicates that Type III verbs, whose associated transfers are non-guaranteed and whose participants are not co-located, have, as Rohde et al. put it "a diminished sense of guaranteed transfer" (2006:698) and indeed pattern with non-ToP verbs, in failing to redirect the hearer's/reader's attention to the recipient.

In addition to looking at ToP verb types, Rohde et al. (2006) also examined COHERENCE RELATIONS. Coherence relations are particular relations that hold between sentences and are used to render an unfolding discourse coherent (Kehler 2002). Specifically, Rohde et al. (2006) examined three types of coherence relations in their pronoun interpretation study: EXPLANATION, PARALLEL, and OCCASION. EXPLANATION relations provide a motivation for the agent's action specified in a prior utterance, as in (4); PARALLEL relations provide points of similarity and contrast among the events being related in a discourse, as in (5); and OCCASION relations provide a point of connection between ordered events, as in (6).

\footnotetext{
${ }^{1}$ The fourth possibility (guaranteed transfer, participants not co-located) does not occur given that a transfer cannot be guaranteed if the participants are not in close physical proximity.

${ }^{2}$ Rohde et al. found this bias to the RECIPIENT argument only for the class of verbs in the perfect; verbs in the imperfective were found to display a bias to the SOURCE argument for all three verb types.
} 
Matt passed a sandwich to David. He didn't want David to starve.

Matt passed a sandwich to David. He put fruit on his plate, too.

Rohde et al. (2006) found that participants disambiguated pronouns differently based on which coherence relation they described in the follow-up sentence that they were asked to produce. Specifically, Rohde et al. found that when participants produced examples of an OCCASION relation in their story completions, the ambiguous pronoun was interpreted as coreferential with the RECIPIENT of the previous sentence and not the SOURCE; no other coherence relation produced such a bias.

More recently, Salomon \& Ward (2016) have shown similar thematic role biases using a reaction time task. Each target stimulus consisted of a ToP verb with SOURCE and RECIPIENT roles filled by discourse referents that are evoked by occupation-denoting NPs, as in (6).

The banker sent a message to the potter. SOURCE RECIPIENT

With ToP verbs, participants reacted significantly faster to lexical associates ${ }^{3}$ of the RECIPIENT occupation than to the SOURCE occupation, presumably reflecting the heightened salience of the former. With non-ToP verbs (e.g. smile at), however, there was no effect. These findings replicate previous studies showing that thematic role information affects the relative salience of discourse entities.

2. Current Study. In the current study, we continue the investigation of whether and to what extent transfer verbs differentially affect the salience of particular thematic roles. Specifically, the goal of this study is two-fold: (a) to replicate the findings of Rohde et al. (2006) using their story completion paradigm and (b) to extend their findings by using a timed lexical decision paradigm. We hypothesize that participants' reaction times to thematic role associates will vary by the type of verb used in that sentence. If the readers' attention has been directed to the RECIPIENT role, readers should be faster to recognize associates related to that role, but slower for words related to the SOURCE. On the other hand, if their attention is directed towards the SOURCE role, they should be faster to recognize associates that correspond to that role, and slower to words related to the RECIPIENT.

To investigate the effect of ToP verbs on thematic role interpretation, we conducted a series of five studies employing two different paradigms: a sentence-completion and a reactiontime task, discussed in turn below. For all studies, participants accessed the surveys and questions via Mechanical Turk and were reimbursed for their time and data. ${ }^{4}$

2.1. SENTENCE COMPLETION STUDIES. These studies were designed to replicate the sentencecompletion study described in Rohde et al. 2008. However, instead of using proper names as verb arguments (as Rohde et al. did; see (3) above), we used occupation-denoting NPs as we did in our earlier study (Salomon \& Ward 2016). We predicted that when presented with sentences

\footnotetext{
${ }^{3}$ Latent Semantic Analysis (LSA) is a database of words gathered by computationally aggregating corpora (Landauer, Foltz \& Laham 1998). The database contains an index of the relative frequency with which a given word appears near a target word. Associates were generated using LSA by entering each unique occupation into the LSA one-to-one matrix, which produced a list of frequently co-occurring associates. For each occupation, one of these associates was selected for inclusion in the study based on the authors' assessment of its suitability. In each case, the associate had at least a .5 ratio to the target occupation.

${ }^{4}$ The survey was created in Qualtrics, an online survey system that records participants' responses.
} 
containing ToP verbs, participants would produce more follow-up sentences with references to the PATIENT argument than they would reference to the SOURCE argument.

2.1.1. EXPERIMENT 1. The goal of Experiment 1 was to establish the viability of our sentencecompletion paradigm that relies on the use of occupation-denoting NPs as opposed to proper names.

2.1.1.1 EXPERIMENT 1: PROCEDURE. In this study, participants were asked to read sentences and create a natural continuation to the sentence they had just read, referencing one of the discourse referents evoked in the previous sentence. For example, a participant would read one of two sentences containing a ToP verb and one of two sentences containing a non-ToP verb, as illustrated in (8a-b) and (9a-b), respectively:

(8) a. The banker transmitted a message to the potter. He/she...

b. The potter transmitted a message to the banker. He/she...

(9) a. The doctor smiled at the ballerina. He/she...

b. The ballerina smiled at the doctor. He/she...

Participants were asked to produce natural continuations of the sentence presented. Each continuation was coded by 2 coders, whose inter-rater reliability exceeded $90 \%$. We expected to replicate the effect that ToP verbs have been shown to have on the relative salience of the thematic roles in question.

2.1.1.2 EXPERIMENT 1: RESULTS. Participants produced significantly more continuations related to the RECIPIENT role $(M=0.72)$ than to the SOURCE role $(M=0.28)$ after being presented with a sentence containing a ToP verb $(t(2854)=26.78, p<0.00)$. However, after being presented with a sentence containing a non-ToP verb, participants produced significantly more continuations related to the AGENT role $(M=0.59)$ than they did to the PATIENT $(M=0.24)$ role $(t(3406)=22.05$, $p<0.00)$. These results are illustrated in the following graph.

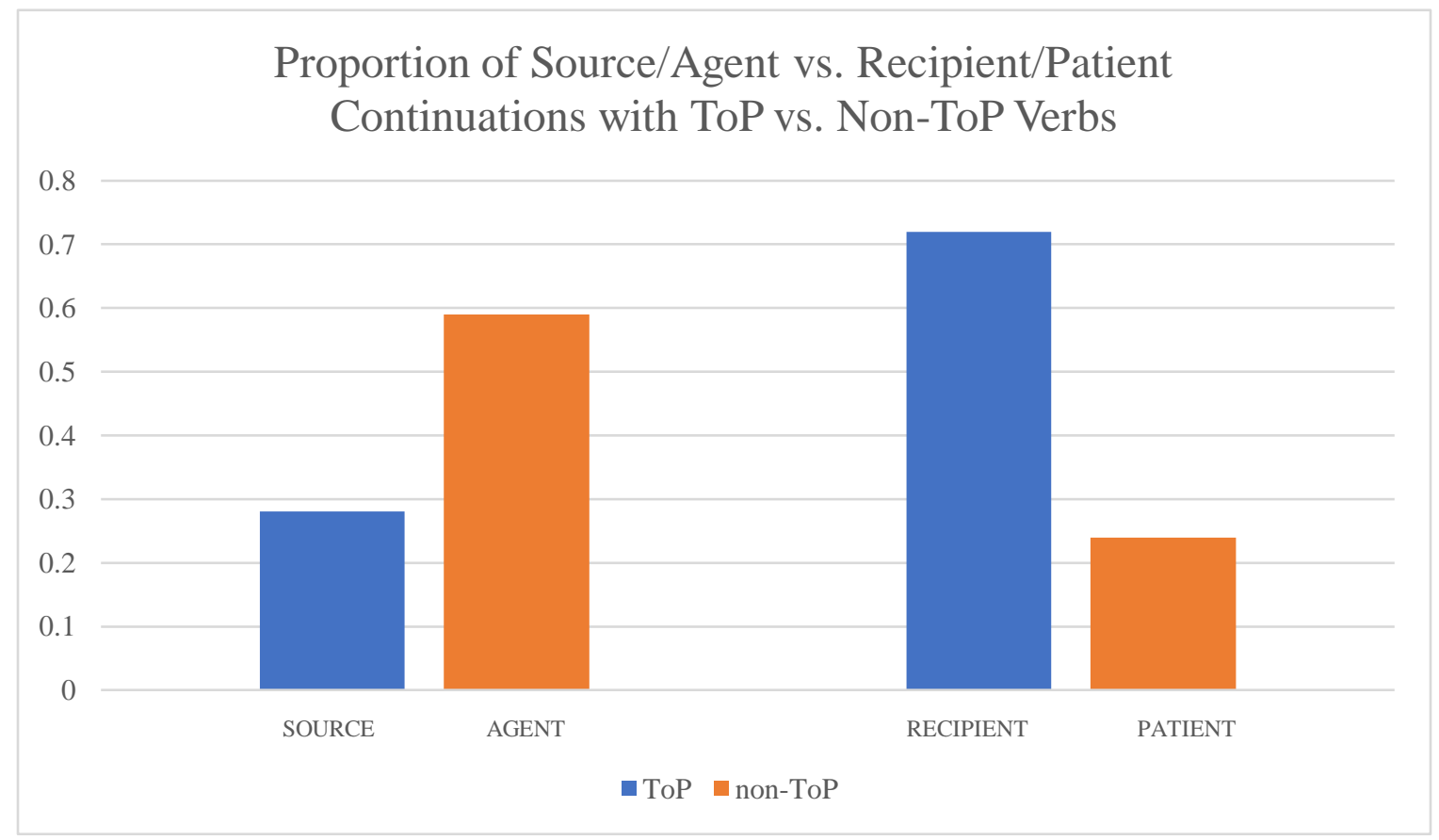


These data replicate earlier studies that showed that ToP verbs redirect a participant's attention to the discourse referent filling the RECIPIENT role of the sentence, who is then more likely to be mentioned as the subject of the subsequent sentence.

2.1.2 SENTENCE COMPLETION STUDY: EXPERIMENT 2. The previous experiment measured the reader's attentional shift towards the RECIPIENT and away from the SOURCE, replicating the ToP verb effect found in Rohde et al. 2006 and Salomon and Ward 2016. In Experiment 2, we investigate the role that the three coherence relations discussed above - EXPLANATION, PARALLEL, or OCCASION - play in the continuations that participants produce. Recall that Rohde et al. (2006) found a bias towards the RECIPIENT of the previous sentence when participants produced examples of an OCCASION relation in their story completions; no other coherence relation produced such a bias.

2.1.2.1 EXPERIMENT 2: PROCEDURE. The procedure followed in Experiment 2 was identical to that of Experiment 1. The only difference was the coding: Each continuation was coded by 2 coders as being one of the EXPLANATION, PARALLEL, or OCCASION RELATIONS. As with Experiment 1, each continuation was coded by 2 coders, whose inter-rater reliability was $89 \%$.

2.1.2.2 EXPERIMENT 2: RESULTS. The majority of sentence completions were coded as providing an EXPLANATION relation (55\%); with an OCCASION relation provided in $20 \%$ of completions, and a PARALLEL relation in $7 \%$ of the responses. ${ }^{5}$ For these three coherence relations produced by the participants, we found significant correlations between the subject of the completed sentence and a particular thematic role. First, we found that when an EXPLANATION coherence relation is provided, there is a significant bias towards the SOURCE $(M=.34, S E=0.03)$ role as compared to the RECIPIENT $(M=0.18, S E=0.03) t(388)=3.79, p<0.00$. The same bias was present even more strongly with PARALLEL coherence relations: every instance in which a PARALLEL coherence relation was provided, we found a concomitant reference to the SOURCE $t(406)=3.87, p<0.00$. Replicating Rohde et al., we find that only when an OCCASION relation is provided do we see a bias towards the RECIPIENT $(M=0.16, S E=0.03)$, rather than to the SOURCE $(M=0.03, S E=0.01)$, $t(406)=4.29, p<0.00$. These data can be summarized as in Tables 2 and 3.

Coherence Relation

EXPLANATION

PARALLEL

OCCASION

Other
Proportion of total continuations

$$
\begin{gathered}
44 \%(\mathrm{n}=22) \\
2 \%(\mathrm{n}=1) \\
44 \%(\mathrm{n}=22) \\
10 \%(\mathrm{n}=5)
\end{gathered}
$$

Table 2: Proportion of total continuations

\begin{tabular}{cccc} 
Coherence Relation & SOURCE & RECIPIENT & Other \\
\hline EXPLANATION $(\mathrm{n}=22)$ & $59 \%(\mathrm{n}=13)$ & $36 \%(\mathrm{n}=8)$ & $5 \%(\mathrm{n}=1)$ \\
PARALLEL $(\mathrm{n}=1)$ & $100 \%(\mathrm{n}=1)$ & $0 \%$ & $0 \%$ \\
\multicolumn{2}{l}{ Of the remaining continuations, $18 \%$ were coded as 'other'. } & &
\end{tabular}




$\begin{array}{cccc}\text { OCCASION }(\mathrm{n}=22) & 9 \%(\mathrm{n}=2) & 86 \%(\mathrm{n}=19) & 5 \%(\mathrm{n}=1) \\ \text { Other }(\mathrm{n}=5) & 40 \%(\mathrm{n}=2) & 20 \%(\mathrm{n}=1) & 40 \%(\mathrm{n}=2)\end{array}$

Table 3: Proportion of references to argument types

2.1.3 SENTENCE COMPLETION STUDY: EXPERIMENT 3. In this experiment, we considered whether and to what extent the type of ToP verb (as described above in §1) affected participants' sentence completions with respect to subsequent references to the SOURCE or RECIPIENT. We hypothesized, based on the earlier findings of Rohde et al., that participants will favor the SOURCE role with Type 3 ToP verbs, with Type 1 and Type 2 ToP verbs showing no such effect.

2.1.3.1 EXPERIMENT 3: PROCEDURE. The procedure used in Experiment 3 was identical to that of Experiment 1. Each sentence continuation was coded by 2 coders, whose inter-rater reliability exceeded $90 \%$.

2.1.3.2 EXPERIMENT 3: RESULTS. Across each of the three ToP verb types, we found no differences in frequency of subsequent references to the SOURCE vs. RECIPIENT argument, contrary to our prediction $(F \mathrm{~s}<2)$. This indicates that the ToP verb type did not bias participants' completions towards either the SOURCE or RECIPIENT. These results are illustrated in the graph below.

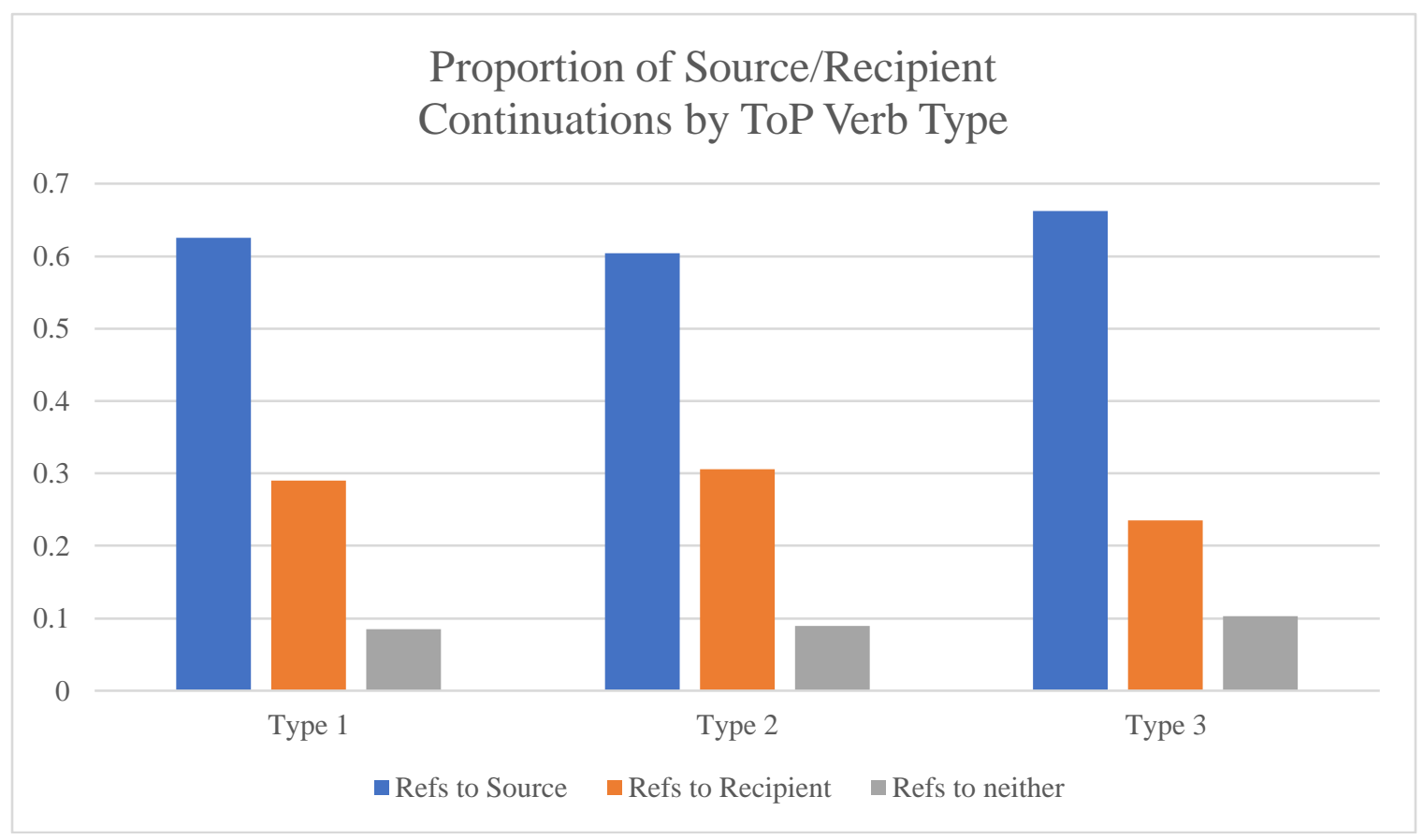

2.1.4 SENTENCE COMPLETION STUDY: DisCUSSION. The data from our three sentence-completion studies suggest that, at least for this paradigm, the effect of discourse-level factors, such as coherence relations, swamp the effect of sentence-level factors, such as ToP verb type. 
2.2 REACTION TIME STUDIES. In the first set of experiments, we asked participants to produce a mini-discourse, illustrating the effects of coherence relations. In the absence of multi-sentence discourses, where the establishment of a coherence relation is not at issue, what can we expect to find? To answer this question, we conducted a series of lexical decision studies, presenting participants with single sentences and measuring their reaction times to lexical associates of the SOURCE and RECIPIENT roles under various conditions.

2.2.1 REACTION TIME STUDY: EXPERIMENT 4 In this study, we investigated participants' reaction times to lexical associates of SOURCE and RECIPIENT arguments across the three ToP verb types discussed above. We hypothesized that the more 'guaranteed' the transfer (in the sense of Rohde et al. 2006), the faster RTs would be to associates of the RECIPIENT argument. Similarly, we hypothesized that the less 'guaranteed' the transfer, the faster RT's would be to associates of the SOURCE argument.

2.2.1.1 EXPERIMENT 4: PROCEDURE The materials used in Experiment 4 were similar to those of the sentence completion experiments (1-3), differing only in the absence of a continuation probe. Participants were asked to read one of two sentences containing a ToP verb, drawn from the same set used in the sentence completion studies. A sample stimulus set is provided in 10(a-b).

(10) a. The banker transmitted a message to the potter.

b. The potter transmitted a message to the banker.

For the lexical decision task, we generated lexical associates for each of the 48 occupationdenoting NPs used in the study. ${ }^{6}$ For example, in (10a), the associate for the SOURCE argument (the banker) was loans and the associate for the RECIPIENT argument (the potter) was clay. In presenting the stimuli, we counterbalanced the materials so participants were equally likely to see the ordering in (10a) as in (10b). Finally, we included 24 5-letter non-words (e.g. snorgs) in the study, all of which were phonologically possible in English. ${ }^{7}$

As with the sentence-completion experiments (1-3), the study was created on Qualtrics and presented to participants on Mechanical Turk. After providing consent, participants were provided with instructions and were told to complete the survey "at their own pace". The instructions indicated participants would be reading sentences about events that occurred on a reality television show and that the sentences would be about contestants on the show.

After reading a sentence, participants were instructed to advance to the next screen, where they were asked to first focus on an asterisk presented on the screen before a string of letters would appear. This string would either be a (real word) associate of the SOURCE argument or a (real word) associate of the RECIPIENT argument, or a non-word (as described above), presented randomly. Participants were then asked to perform a lexical decision task (LDT) in which they had to decide whether the string of letters on the screen were a common English word or not. They were asked to indicate their response by clicking 'Yes' or 'No' on the screen and to do so "as accurately and as quickly as possible". Qualtrics was programmed to record the

\footnotetext{
${ }^{6}$ As in our previous study (Salomon \& Ward 2016), these associates were generated via Latent Semantic Analysis (LSA).

${ }^{7}$ These 5-letter words were generated through the Australian Research Council (ARC) non-word data base (Rastle, Harrington, and Coltheart 2002).
} 
participants' responses and the time between word presentation and response. Reaction times for the LDT were measured as the primary dependent measure. ${ }^{8}$

2.2.1.2 EXPERIMENT 4: RESULTS. As hypothesized, we find that participants' RTs to associates of the SOURCE and RECIPIENT arguments are affected by the degree to which the transfer event is 'guaranteed'. While we found no such effect in our sentence completion study (Experiment 3), here we find a significant effect of ToP verb type on participants' RTs. Participants respond faster to the RECIPIENT $(M=1411 \mathrm{~ms}, S E=8 \mathrm{~ms})$ than the SOURCE $(M=1464, S E=8)$ for Type 1 transfer verbs $(t(5848)=4.72, p<0.00)$. Recall that Type 1 is the ToP verb type in which transfer is considered most guaranteed and, as such, RTs to the RECIPIENT are facilitated. However, with Type 3 ToP verbs, RTs to associates of the SOURCE argument are actually facilitated $(M=1443$, $S E=10)$ as compared to those of the RECIPIENT argument $(M=1468, S E=20)(t(1918)=2.18$, $p=0.029$ ). These results are summarized in the following graph.

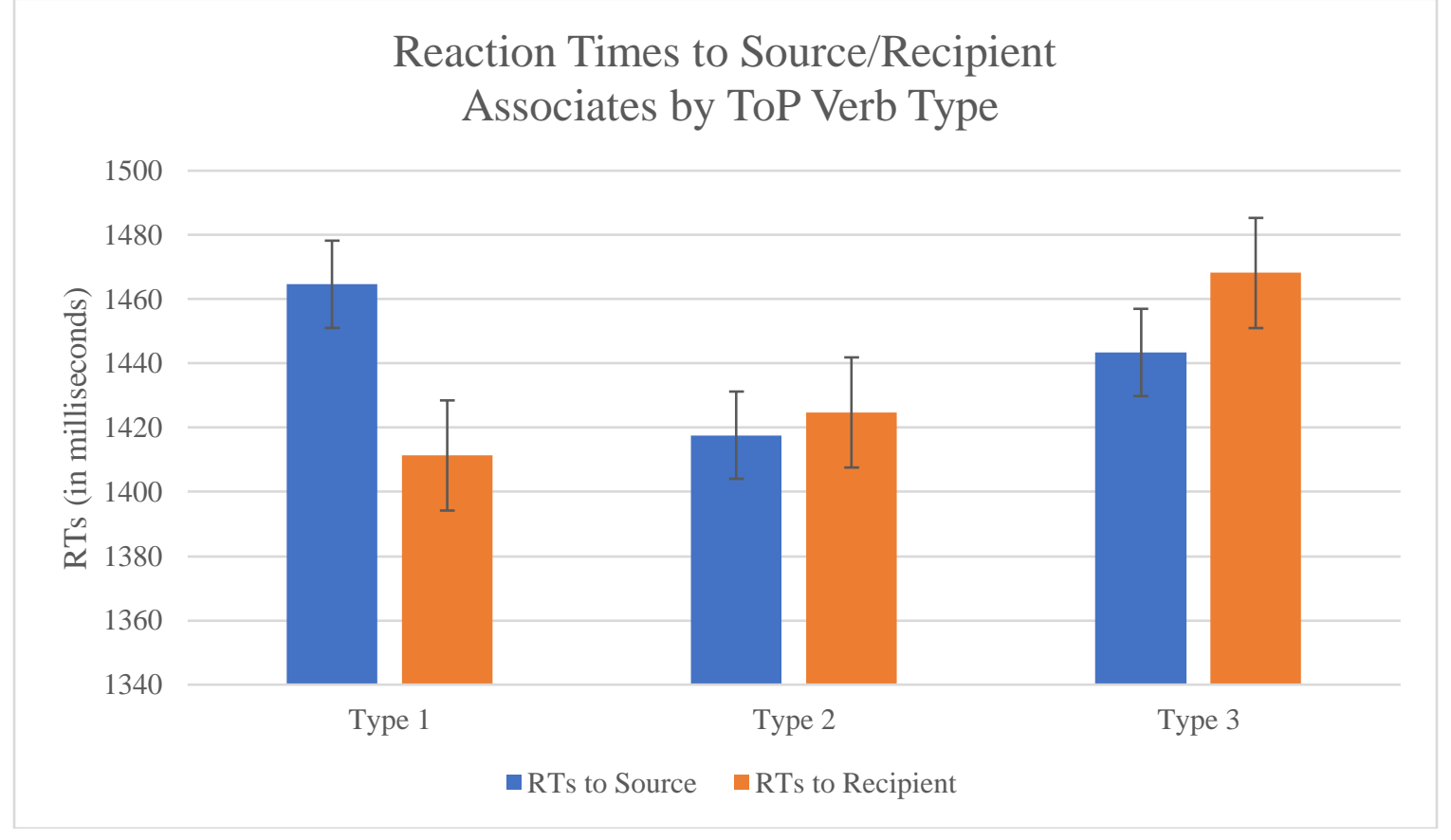

2.2.2 REACTION TIME STUDY: EXPERIMENT 5. In this study, we consider the effect of semantic environment on RTs. Using the methodology of Salomon \& Ward (2016), we presented participants with transfer verbs in seven different semantic environments: positive and negative factives, futures, hypotheticals (It'd be awesome if the banker...); propositional attitudes (The banker considered...), sentential negation (The banker didn't...), and incorporated negation (The banker failed to...). We hypothesized that in single-sentence discourses, participants will be sensitive to whether the transfer event has been asserted or presupposed to have actually occurred in the discourse provided; that is, whether the event is REALIZED or UNREALIZED. Given previous findings that participants' attention seemed to shift following an event depicted by a transfer verb, we hypothesized that different semantic environments would

\footnotetext{
${ }^{8}$ Occasionally, after being presented with a sentence, participants were asked to complete a simple text comprehension question, to ensure attentiveness to the task. The question probed about the facts presented in the previous sentence, and participants had to respond 'Yes' or 'No'.
} 
differentially affect this attentional shift. For example, factive environments, which presuppose a realized transfer, should facilitate RTs to associates of RECIPIENT arguments. Consider (11):

(11) The banker resented giving a sweater to the potter.

Here, we hypothesized that RTs to associates of potter would be faster with respect to those of banker, replicating our earlier finding with respect to non-factive environments. Moreover, we predict this facilitation with presupposed realized transfers whether the affect of the factive is positive or negative, as illustrated in 12(a-b), respectively:

(12) a. The banker relished giving a sweater to the potter.

b. The banker resented giving a sweater to the potter.

On the other hand, negative environments, as illustrated in (13), which deny a realized transfer, should facilitate RTs to SOURCE arguments.

(13) The banker didn't give a sweater to the potter.

Considering a broad range of semantic environments, we distinguished among those that we hypothesized would facilitate faster RTs to the RECIPIENT argument from those that we hypothesized would not, based on the extent to which the event is (un)realized.

\section{REALIZED EVENTS}

Simple

Factive (Positive Affect)

Factive (Negative Affect)
The banker gave a sweater to the potter.

The banker relished giving a sweater to the potter.

The banker resented giving a sweater to the potter. UNREALIZED EVENTS

Future The banker will give a sweater to the potter.

Hypothetical

Negative

Propositional Attitude
It would be great if the banker gave a sweater to the potter.

The banker failed to give a sweater to the potter.

The banker considered giving a sweater to the potter.

Table 4: Realized and Unrealized Semantic Environments

Overall, we predicted that environments in which the event is REALIZED would facilitate RTs to associates of RECIPIENT arguments, whereas environments in which the event is UNREALIZED would facilitate RTs to those of SOURCE arguments, in that the participant's attention should remain with the SOURCE.

2.2.2.1 EXPERIMENT 5: PROCEDURE. The procedure used in Experiment 5 was identical to that of Experiment 4.

2.2.2.2 EXPERIMENT 5: RESULTS. Inconsistent with our hypothesis, the data do not support a distinction based on whether the transfer event is REALIZED or UNREALIZED; in fact, no such difference was found $(F(1,907)=.92, p=.34)$. For example, we found that RTs to associates of SOURCE arguments (vs. RECIPIENT arguments) were significantly faster in positive factive 
environments (12a), even though the transfer is semantically presupposed to have occurred. However, RTs to associates of SOURCE arguments in negative factive environments (12b) were significantly slower $(F(1,791)=9.54, p<.002)$ that RECIPIENT arguments. Conversely, RTs in negative environments $(13 \mathrm{c})$ to RECIPIENT probes were significantly faster than to SOURCE probes, even though no transfer event is realized $(F(1,83=7.43, p<.008)$.

2.2.2.3 EXPERIMENT 5: DISCUSSION. Given that RTs did not vary based on whether the event was realized or not, the data suggest a different account. We suggest alternatively that the semantic environments described in Table 4 are directing participants to take a particular perspective on the event described. This perspective may be associated with one of the thematic roles represented in the event, specifically the SOURCE or the RECIPIENT role, and, as a result, greater salience is predicted to be associated with that thematic role. Thus, the perspective on the event should drive the relative salience of the participating thematic roles. We shall refer to this possibility as the Perspectival Hypothesis. We propose three perspectives that participants can take when processing a discourse: a SOURCE-oriented perspective, a RECIPIENT-oriented perspective, or a neutral perspective, external to the event depicted.

SOURCE-oriented perspectives are associated with those events whose explanation lies with the SOURCE, on which the participant's attention can be expected to remain (Kehler 2002). Such perspectives may provide a SOURCE-centric explanation for the event in question, in that it sheds light on the emotional state of the SOURCE of the sentence. Examples of SOURCE-oriented perspectives are propositional attitudes (e.g., The banker feared...) and positive factives (e.g. The banker relished...).

RECIPIENT-oriented perspectives, on the other hand, are associated with those events whose explanation lies with the RECIPIENT, to which the participant's attention can be expected to shift. Such perspectives may provide a RECIPIENT-centric explanation for the event in question, in that it sheds light on the emotional state of the RECIPIENT of the sentence. Examples of RECIPIENToriented perspectives are negative factives (e.g., The banker resented...) and incorporated negations (The banker refused to...).

Finally, an external perspective is a neutral one in that it does not provide an internal (SOURCE or RECIPIENT) perspective. Examples of external perspectives are simple preterites (e.g. The banker...), futures (e.g. The banker will...), or hypotheticals (e.g. It would be great if the banker...). For events with a neutral (or external) perspective, we hypothesize that participants' attention is free to shift to the RECIPIENT, replicating earlier findings.

To test the Perspectival Hypothesis, we coded each semantic environment as being one of three perspectives and reanalyzed the data. We found that participants are indeed aware of these perspectives, as measured by their reaction times. Participants respond faster to words related to the SOURCE argument than to the RECIPIENT argument for the SOURCE-oriented perspective environments than for neutral environments $(F(1,3892)=5.46, p=.019)$. Furthermore, participants respond faster to words related to the RECIPIENT argument than to the SOURCE argument for the RECIPIENT-oriented perspective environments $(t(867)=3.50, p=0.0005)$.

It should be noted that similar semantic environments had significantly different effects on RTs to the SOURCE/RECIPIENT. For example, the class of factive environments produced vastly different RTs: positive factive produced a bias towards the SOURCE argument, while a negative factive environment produce a bias towards the RECIPIENT argument $(F(1,791)=9.54, p<0.002)$. 
Such a result is surprising and indicates that participants are sensitive to subtle differences even within rather similar semantic environments. ${ }^{9}$

3. General Discussion \& Summary. We find that the two paradigms that we employed in these five experiments differentially highlight participants' sensitivity to various features of discourses featuring ToP verbs. When a mini-discourse is elicited in a sentence completion task, participants posit a particular coherence relation, which in turn affects whether the SOURCE or RECIPIENT discourse entity is referenced (Rohde, Kehler, and Elman 2006). However, in an RT task, when participants consider sentences in isolation and without having to specify coherence relations, they rely more on the lexical properties of the transfer verb itself.

In sum, our RT study - being a more automatic task - is arguably more sensitive to sentence-level linguistic features of the relevant event (e.g., ToP verb type). The sentence completion task, on the other hand, is a more strategic task in which participants are guided by discourse-level features of the relevant event (e.g., coherence relations). In conclusion, our findings support the notion that coherence relations drive the interpretation of multi-sentence discourses. However, when sentences are considered in isolation, participants must rely instead on particular features of the linguistic context.

\section{References:}

Bolinger, Dwight. 1975. Aspects of language, $2^{\text {nd }}$ edition. New York: Harcourt Brace Jovanovich.

Crawley, Rosalind A., Rosemary J. Stevenson, and David Kleinman. 1990. The use of heuristic strategies in the interpretation of pronouns. Journal of Psycholinguistic Research 19(4). 245-264.

Ferretti, Todd R., Hannah Rohde, Andrew Kehler, and Melanie Crutchley. 2009. Verb aspect, event structure, and coreferential processing. Journal of Memory and Language 61(2). 191-205. https://doi.org/10.1016/j.jml.2009.04.001.

Gernsbacher, Morton Ann, and David J. Hargreaves. 1988. Accessing sentence participants: the advantage of first mention. Journal of Memory and Language 27(6). 699-717. https://doi.org/10.1016/0749-596X(88)90016-2.

Graesser, Arthur C., Murray Singer, and Tom Trabasso. 1994. Constructing inferences during narrative text comprehension. Psychological Review 101(3). 371-395.

Grice, H. P. 1975. Logic and conversation. In Peter Cole \& Jerry L. Morgan (eds.), Syntax and Semantics, Volume 3: Speech Acts. 41-58. New York: Academic Press.

Kehler, Andrew. 2002. Coherence, Reference, and the Theory of Grammar. CSLI publications Stanford, CA. http://web.stanford.edu/group/cslipublications/cslipublications/pdf/1575862166.pdf.

Kintsch, Walter. 1994. Text comprehension, memory, and learning. American Psychologist 49 (4). 294-303.

Kintsch, Walter, and Teun A. Van Dijk. 1978. Toward a model of text comprehension and production. Psychological Review 85(5). 363-394.

\footnotetext{
${ }^{9}$ It may be that effects we found for the valenced factives (positive and negative) may be the result of the presence of implicit causality verbs within our stimuli (Andy Kehler, p.c.). We are currently investigating whether implicit causality alone is driving these effects.
} 
Koornneef, Arnout W., and Jos J.A. Van Berkum. 2006. On the use of verb-based implicit causality in sentence comprehension: Evidence from self-paced reading and eye tracking. Journal of Memory and Language 54(4). 445-465. doi: 10.1016/j.jml.2005.12.003

Landauer, Thomas K., Peter W. Foltz, and Darrell Laham. 1998. An introduction to latent semantic analysis. Discourse Processes 25(2-3). 259284. https://doi.org/10.1080/01638539809545028.

Rastle, Kathleen, Jonathan Harrington, and Max Coltheart. 2002. 358,534 nonwords: The ARC nonword database. The Quarterly Journal of Experimental Psychology: Section A 55(4). 1339-1362. https://doi.org/10.1080/02724980244000099.

Rohde, Hannah, Andrew Kehler, and Jeffrey L. Elman. 2006. Event structure and discourse coherence biases in pronoun interpretation. In Proceedings of the 28th Annual Conference of the Cognitive Science Society. 697-702. http://www.lel.ed.ac.uk/ hrohde/papers/RohdeKehlerElman.cogsci.2006.pdf.

Salomon, Meghan M. \& Gregory Ward. 2016. The effect of perfective aspect on the salience of discourse entities. Poster presented at the 29th Annual Meeting of the CUNY Conference on Human Sentence Processing, Gainesville, FL.

Whorf, Benjamin L. 1956. Language, Thought and Reality: Selected Writings of Benjamin Lee Whorf. Edited by John B. Carroll. Cambridge, MA: MIT Press.

Wunderlich, Dieter. 2006. Towards a structural typology of verb classes. Advances in the theory of the lexicon. Berlin: Mouton de Gruyter: 58-166. 